\title{
Flux-Limited Diffusion Approximation Models of Giant Planet Formation by Disk Instability
}

\author{
Alan P. Boss \\ Department of Terrestrial Magnetism, Carnegie Institution of Washington, 5241 Broad \\ Branch Road, NW, Washington, DC 20015-1305 \\ boss@dtm.ciw.edu
}

\begin{abstract}
Both core accretion and disk instability appear to be required as formation mechanisms in order to explain the entire range of giant planets found in extrasolar planetary systems. Disk instability is based on the formation of clumps in a marginally-gravitationally unstable protoplanetary disk. These clumps can only be expected to contract and survive to become protoplanets if they are able to lose thermal energy through a combination of convection and radiative cooling. Here we present several new three dimensional, radiative hydrodynamics models of self-gravitating protoplanetary disks, where radiative transfer is handled in the flux-limited diffusion approximation. We show that while the flux-limited models lead to higher midplane temperatures than in a diffusion approximation model without the flux-limiter, the difference in temperatures does not appear to be sufficiently high to have any significant effect on the formation of self-gravitating clumps. Self-gravitating clumps form rapidly in the models both with and without the flux-limiter. These models suggest that the reason for the different outcomes of numerical models of disk instability by different groups cannot be attributed solely to the handling of radiative transfer, but rather appears to be caused by a range of numerical effects and assumptions. Given the observational imperative to have disk instability form at least some extrasolar planets, these models imply that disk instability remains as a viable giant planet formation mechanism.
\end{abstract}

Subject headings: accretion, accretion disks - hydrodynamics - instabilities planetary systems: formation - solar system: formation 


\section{Introduction}

Observations of protoplanetary disks around $\mathrm{T}$ Tauri stars traditionally imply disk masses in the range of 0.01 to $0.1 M_{\odot}$ (Kitamura et al. 2002). However, these disk masses may well be underestimated by as much as a factor of 10 (Andrews \& Williams 2007). In addition, young stellar objects are likely to have had even higher disk masses at ages younger than typical T Tauri stars (a few Myr), as their protostellar disks transitioned into protoplanetary disks. Combined with the need for planet formation theorists to prefer increasingly higher disk masses in order to account for the timely formation of gas giants by core accretion (e.g., Inaba et al. 2003 suggest a $0.08 M_{\odot}$ disk, while Alibert et al. 2005 considered disks as massive as $0.1 M_{\odot}$ ), it is becoming clear that at least some protoplanetary disks are likely to have experienced a phase of gravitational instability, which might have led to the rapid formation of gas giant planets by the disk instability mechanism (e.g., Boss 1997; Mayer et al. 2002). The absence of IR excesses in $\sim 65 \%$ of the youngest stars observed by Spitzer suggests that the majority of protoplanetary disks dissipate on time scales of $\sim 1$ Myr or less (Cieza et al. 2007). While core accretion models can be constructed that permit giant planet formation times less than 1 Myr (Chambers 2006), other assumptions can require formation times of several Myr (e.g., Inaba et al. 2003; Alibert et al. 2005).

Considering that estimates of the frequency of gas giant planets around $\mathrm{G}$ dwarfs with orbits inside $\sim 20$ AU range from $\sim 20 \%$ to $\sim 40 \%$, there is a need for at least one robust formation mechanism for gas giant planets. It is important to note that both core accretion and disk instability appear to be needed to explain the range of extrasolar planets detected to date. Core accretion would seem to be the preferred mechanism to form giant planets with very large inferred core masses. E.g., HD 149026 b has been inferred to have a core mass of $70 M_{\oplus}$ with a gaseous envelope of $\sim 40 M_{\oplus}$ (Sato et al. 2005), though the formation of this planet is hard to explain even by core accretion (Ikoma et al. 2006). Disk instability would seem to be the preferred mechanism for forming gas giants in very low metallicity systems (e.g., the M4 pulsar planet, where the metallicity [Fe/H] =-1.5 [Sigurdsson et al. 2003], and perhaps the giant planets orbiting HD 155358 and HD 47536, both of which have $[\mathrm{Fe} / \mathrm{H}]=$ -0.68 [Cochran et al. 2007]). While disk instability is somewhat insensitive to metallicity (Boss 2002), recent models have suggested that higher metallicity could aid in the formation of giant planets by disk instability (Mayer et al. 2007). Disk instability also appears to be needed to form gas giant planets around M dwarf stars (Boss 2006b), though the situation regarding formation by core accretion is unclear at present (Laughlin et al. 2004; cf., Kornet et al. 2006).

Based on all of these observations and detections, then, the main theoretical questions

would seem to be whether there is a formation mechanism that can account for the majority of 
extrasolar planets, and if so, which mechanism it is. In order to answer these questions, theorists have been busily examining core accretion and disk instability in increasingly greater detail. Core accretion has been subjected to considerably greater scrutiny than disk instability, given that it has been the generally accepted mechanism for giant planet formation for almost three decades, dating back to Mizuno (1980), whereas disk instability is only a decade-old as a serious alternative to core accretion (Boss 1997), and is just now beginning to be investigated sufficiently to discover its strengths and weaknesses.

Theorists studying disk instability are divided into two distinct camps, those whose numerical models support the possibility of forming giant planets by this means (e.g., Boss 1997, 2000, 2001, 2002, 2004, 2005, 2006a,b, 2007; Mayer et al. 2002, 2004, 2007), and those whose numerical models (e.g., Pickett et al. 2000; Cai et al. 2006; Boley et al. 2006, 2007a,b) or analytical arguments (e.g., Rafikov 2007) do not. The reason for this basic difference in outcomes is presently unclear, and may be a combination of many effects (Nelson 2006; Boss 2007), such as numerical spatial resolution, gravitational potential solver accuracy, use of artificial viscosity, degree of stellar irradiation, detailed radiative transfer effects, and spurious numerical heating.

Recently attention has been focused on the role of radiative losses from the surface of the disk. A disk instability is likely to be stifled if the optically-thick clumps that form are unable to lose at least some of the thermal energy produced by compressional heating during contraction to protoplanetary densities. While vertical convection appears to be able to cool the disk midplane (Boss 2004; Boley et al. 2006; Mayer et al. 2007; Rafikov 2007), this thermal energy must eventually be radiated away at the disk's surface. Models employing the flux-limited diffusion approximation have been presented by Boley et al. (2006, 2007b) and Mayer et al. (2007), reaching opposite conclusions regarding the possibility of disk instability forming protoplanets. The treatment of radiative boundary conditions for the disk differs for each group. Boley et al. (2006) fit an atmosphere to the flux originating from the interior of the disk. Mayer et al. (2007) assume blackbody emission at the disk surface (for particles defined as being on the surface), while the present models use an envelope bath with a fixed temperature, typically $50 \mathrm{~K}$.

With the exception of a single test model mentioned in passing by Boss (2001), all of the author's disk instability models since Boss (2001) have employed diffusion approximation radiative transfer without a flux-limiter, for reasons of computational performance. We present here three new models that explore in some detail the effects of including a fluxlimiter in disk instability models, in the hopes of helping to decide if this particular numerical choice is responsible for the distinct disparity in outcomes of disk instability models. 


\section{Numerical Methods}

The calculations were performed with a code that solves the three dimensional equations of hydrodynamics and radiative transfer, as well as the Poisson equation for the gravitational potential. This code has been used in all of the author's previous studies of disk instability, and is second-order-accurate in both space and time (Boss \& Myhill 1992).

The equations are solved on a spherical coordinate grid with $N_{r}=101, N_{\theta}=23$ in $\pi / 2 \geq \theta \geq 0$, and $N_{\phi}=512$. The radial grid is uniformly spaced with $\Delta r=0.16 \mathrm{AU}$ between 4 and $20 \mathrm{AU}$. The $\theta$ grid is compressed into the midplane to ensure adequate vertical resolution $\left(\Delta \theta=0.3^{\circ}\right.$ at the midplane). The $\phi$ grid is uniformly spaced, and the central protostar is assumed to move in such a way as to preserve the location of the center of mass of the entire system. The number of terms in the spherical harmonic expansion for the gravitational potential of the disk is $N_{Y l m}=48$. The Jeans length criterion is monitored to ensure that numerical artifacts do not form.

The boundary conditions are chosen at both 4 and 20 AU to absorb radial velocity perturbations. Mass and momentum that enters the innermost shell of cells at 4 AU are added to the central protostar and so removed from the hydrodynamical grid, whereas mass and momentum that reach the outermost shell of cells at 20 AU piles up at the boundary.

\section{Flux-Limited Diffusion Approximation}

All of the author's disk instability models since Boss (2001) have employed radiative transfer in the diffusion approximation, through the solution of the equation determining the evolution of the specific internal energy $E$ :

$$
\frac{\partial(\rho E)}{\partial t}+\nabla \cdot(\rho E \mathbf{v})=-p \nabla \cdot \mathbf{v}+\nabla \cdot\left[\frac{4}{3 \kappa \rho} \nabla\left(\sigma T^{4}\right)\right]
$$

where $\rho$ is the total gas and dust mass density, $t$ is time, $\mathbf{v}$ is the velocity of the gas and dust (considered to be a single fluid), $p$ is the gas pressure, $\kappa$ is the Rosseland mean opacity, $\sigma$ is the Stefan-Boltzmann constant, and $T$ is the gas and dust temperature. The energy equation is solved explicitly in conservation law form, as are the four other hydrodynamic equations.

The final term in the energy equation represents the transfer of energy by radiation in the diffusion approximation, which is valid in optically thick regions of the disk. Given typical midplane optical depths of $\sim 10^{4}$, the diffusion approximation should be valid at the 
disk midplane and throughout most of the disk, though it will break down at the surface of the disk. In order to ensure that the diffusion approximation did not affect the solution in regions where it is not valid, Boss (2001) used a simple artifice to control the flux in the low optical depth regions of the disk: the divergence of the radiative flux term was set equal to zero in regions wherever the optical depth $\tau$ dropped below a critical value $\tau_{\text {crit }}$, where $\tau_{\text {crit }}$ was typically set equal to 10 .

An alternative approach to treating the low optical depth regions of disks in the diffusion approximation is to employ a flux-limiter (e.g., Bodenheimer et al. 1990). The purpose of a flux-limiter is to enforce the physical law that in low optical depth regions the ratio of the radiative flux $\vec{F}$ to the radiative energy density $e_{r}$ cannot exceed the speed of light $c$, i.e., $|\vec{F}| \leq c e_{r}$. Bodenheimer et al. (1990) adopted a prescription for enforcing this constraint based on the flux-limiter proposed by Levermore \& Pomraning (1981) for the situation where scattering of light is negligible. The Levermore \& Pomraning (1981) flux-limiter is based on a heuristic argument leading to an approximation consisting of a rational function that uses a polynomial involving gradients of the radiation energy density. They then tested their formulation against an exact solution for planar geometry, i.e., one-dimensional radiative transfer. Their flux-limiter has been employed by Boley et al. (2006) and by Mayer et al. (2007) in their disk instability calculations, with differing results, as well as in the molecular cloud collapse models of Whitehouse \& Bate (2006).

The author's diffusion approximation code is derived from a code that handles radiation transfer in the Eddington approximation (Boss 1984; Boss \& Myhill 1992). In this code, the energy equation is solved along with the mean intensity equation, given by

$$
\frac{1}{3} \frac{1}{\kappa \rho} \nabla \cdot\left(\frac{1}{\kappa \rho} \nabla J\right)-J=-B
$$

where $J$ is the mean intensity and $B$ is the Planck function $\left(B=\sigma T^{4} / \pi\right)$. The mean intensity $J$ is related to the radiative energy density $e_{r}$ by $J=c e_{r} / 4 \pi$, while the net flux vector $\vec{H}$ is given by $\vec{H}=\vec{F} / 4 \pi$. Hence, the statement of physical causality $|\vec{F}| \leq c e_{r}$ is equivalent to $|\vec{H}| \leq J$. The Eddington approximation version of the code does not calculate $\vec{H}$ directly, but rather $\nabla \cdot \vec{H}$, as this quantity is used in the code to calculate the time rate of change of energy per unit volume due to radiative transfer, $L$, through

$$
L=-4 \pi \nabla \cdot \vec{H}=\frac{4 \pi}{3} \nabla \cdot\left(\frac{1}{\kappa \rho} \nabla J\right)
$$

in optically thick regions (Boss 1984). Hence, it is convenient to apply the physical causality constraint $|\vec{H}| \leq J$ in another form. Using the equation for $L$, one finds 


$$
\vec{H}=-\frac{1}{3 \kappa \rho} \nabla J
$$

The constraint $|\vec{H}| \leq J$ then becomes

$$
\left|\frac{4 \pi}{3 \kappa \rho} \nabla J\right| \leq 4 \pi J
$$

This constraint is then evaluated in a convenient but approximate manner by effectively taking the divergence of both sides of this equation, resulting in a constraint on $L$ that

$$
|L|=\left|\frac{4 \pi}{3} \nabla \cdot\left(\frac{1}{\kappa \rho} \nabla J\right)\right| \leq|4 \pi \nabla \cdot \vec{J}|
$$

where $\vec{J}$ is a pseudovector with $J$ as components in all three directions. In the diffusion approximation, $J=B$. In practice, then, $L$ is calculated for each numerical grid point, and if $|L|$ exceeds $|4 \pi \nabla \cdot \vec{J}|, L$ is set equal to $|4 \pi \nabla \cdot \vec{J}|$ but with the original sign of $L$ (i.e., preserving the sense of whether the grid cell is gaining or losing energy through radiative transfer).

Boss (2001) noted in passing that a model where this flux limiter was employed did not result in any major changes in the progress of the disk instability models under investigation, but provided no details or justification for this statement. The main purpose of this paper is to return to this potentially key point, calculate several new models with this version of flux-limited diffusion, and compare them to a disk instability model without a flux-limiter.

\section{Results}

We now present the results of a set of three new models employing the flux-limiter defined in the previous section. The three models vary only in the value chosen for the critical optical depth $\tau_{\text {crit }}$, below which the term calculating the time rate of change of energy per unit volume due to radiative transfer, $L$ (effectively the divergence of the radiative flux), was set equal to zero. The three models employed $\tau_{\text {crit }}=0.1,1.0,10.0$. In practice, all three of these models evolved in very much the same manner, so figures will only be shown for the model with $\tau_{\text {crit }}=1.0$, termed model FL1. The three models are all continuations in time of model HR of Boss (2001), starting at a time of 322 yrs of evolution in model $\mathrm{HR}$, and continuing for up to another 8 yrs of evolution $(\sim 1 / 2$ clump orbital period). The new models are compared to model TE of Boss (2007), which used diffusion approximation 
radiative transfer, but without the flux-limiter, and which also started from model HR of Boss (2001) after 322 yrs of evolution.

The results for models FL1 and TE at a time of 326 yrs of evolution are shown in Figures 1 and 2. The midplane density contours for the two models are very similar, especially so for the highest density regions (cross-hatched). The densest spiral arms and clumps that exist at this phase of the evolution are located between 6 o'clock and 8 o'clock in Figures 1 and 2 , with maximum densities of $\sim 1.6 \times 10^{-9} \mathrm{~g} \mathrm{~cm}^{-3}$ occurring in the clumps at 6:30 o'clock.

Figures 3 and 4 depict the temperature and optical depth profiles as a function of vertical height above the midplane, along the $\theta$ coordinate direction, starting from the cells with the maximum densities in models FL1 and TE. Two different evaluations of the optical depth are plotted, namely the optical depth in the radial direction (the value used in evaluating all radiative transfer effects, including $L$ and $\tau_{c r i t}$, in model $\mathrm{TE}$ and all previous models by the author, including the flux-limiter test mentioned by Boss 2001), and the optical depth in the $\theta$ direction, which was used for evaluating $L$ and $\tau_{\text {crit }}$ in the three new models. The decision of using an optical depth $\tau$ dependent only on the radial coordinate direction was originally made in order to enforce consistency with spherically symmetric calculations of protostellar cloud collapse, the problem that initially motivated the development and testing of this radiative hydrodynamics code (e.g., Boss \& Myhill 1992; Myhill \& Boss 1993). Figures 3 and 4 show that these two different evaluations of $\tau$ do not differ greatly from each other, varying by no more than a factor of 6 at the same vertical height. Given the spatial resolution in the $\theta$ coordinate, the differences in the two evaluations of where $\tau=\tau_{\text {crit }}$ typically differ by less than one vertical cell. Improving this treatment of $\tau$ in a three dimensional code may require the use of an angle-dependent ray-tracing radiative transfer routine, which would be prohibitively computationally expensive. Alternatively, one could imagine using a weighted mean $\tau$ derived from the values of $\tau$ in the three coordinate directions. Nevertheless, it is apparent from Figures 3 and 4 that the surface of the disk, defined as where $\tau \sim 2 / 3$, falls at a vertical height of $\sim 1.6$ to $1.7 \mathrm{AU}$ in both models.

Figures 5 and 6 are perhaps the most important for discerning the effects of the fluxlimiter, showing the vertical temperature profiles over the maximum density clumps in Figures 1 and 2 for models FL1 and TE. While the temperature differences are hard to discern when plotted on a log scale (Figure 3 and 4), on a linear scale it is clear that in the fluxlimiter model (FL1), there is a much steeper vertical temperature gradient near the surface of the disk than in the model without the flux-limiter (TE), as might be expected. [Note that in both models, the temperature is assumed to fall to $50 \mathrm{~K}$ in the disk's envelope (e.g., Chick \& Cassen 1997).] In spite of this steeper rise below the disk's surface, however, in both models FL1 and TE the profile flattens out near the disk midplane and approaches es- 
sentially the same value of $\approx 100 \mathrm{~K}$, with model FL1 being less than $2 \mathrm{~K}$ hotter than model $\mathrm{TE}$ at the midplane. This similarity in thermal behavior is consistent with the similarities in the density evolution seen in Figure 1 and 2.

The models assume that the disk is immersed in an envelope bath at $50 \mathrm{~K}$. The specific internal energy of the envelope gas in cells with densities less than $10^{-12} \mathrm{~g} \mathrm{~cm}^{-3}$ is recalculated each time step from the internal energy equation of state, using the assumed envelope temperature of $50 \mathrm{~K}$ and the envelope density at each grid cell. The specific internal energy is thus forced to track the temperature profiles displayed in Figures 5 and 6 and so to merge smoothly with the assumed envelope thermal bath. This assumption can lead to either the gain or the loss of internal energy, depending on whether the envelope cell had a temperature lower than or greater than $50 \mathrm{~K}$ before the envelope temperature constraint was applied. It is important to note that while the handling of the disk's surface is directly linked to the ability of the disk to cool itself by radiation into the infalling envelope, this surface treatment has relatively little effect on the cooling of the midplane by convective-like motions, as the driver for these motions is the vertical temperature gradient near the disk's midplane, not the disk's surface. Figure 5 in Boss (2004) shows that the regions of convective instability according to the Schwarzschild criterion are concentrated near the disk midplane, in spite of the fact that the midplane is forced to be convectively stable by the assumption of equatorial reflection symmetry.

Figure 7 and 8 display the results of both models after another 4 yrs of evolution, at $\sim$ 330 yrs, the maximum time to which model FL1 was evolved. It is evident again from these figures that the models continued to evolve in a highly similar manner. In order to quantify this, the dense clumps seen at 7:30 o'clock in Figures 7 and 8 were evaluated in detail. For model FL1, this clump had a maximum density of $1.2 \times 10^{-9} \mathrm{~g} \mathrm{~cm}^{-3}$, and contained a mass of $0.24 M_{\text {Jupiter }}$ within regions with a density no less than $1 / 30$ of the maximum density. This mass exceeds the Jeans mass of $0.23 M_{\text {Jupiter }}$ for this clump, implying that it is gravitationally bound. The ratio of thermal energy to gravitational energy for the clump is 0.84 . The equivalent spherical radius of the clump was $0.38 \mathrm{AU}$, which is smaller than the critical tidal radius of $0.49 \mathrm{AU}$, implying stability against tidal forces. For comparison, the corresponding clump in model TE had a maximum density of $1.5 \times 10^{-9} \mathrm{~g} \mathrm{~cm}^{-3}$, containing a mass of $0.30 M_{\text {Jupiter }}$, compared to a Jeans mass of 0.24 . The ratio of thermal energy to gravitational energy for this clump is 0.77 . The equivalent spherical radius of this clump was $0.39 \mathrm{AU}$, also smaller than the critical tidal radius of $0.52 \mathrm{AU}$. While model TE yielded a clump at this time that was $25 \%$ more massive than in model FL1, both models produced apparently self-gravitating clumps that could go on to form gas giant protoplanets. The estimated orbital eccentricities and semimajor axes are 0.033 and $11.3 \mathrm{AU}$ for the clump in model FL1 and 0.004 and 11.3 for model TE clump at $~ 330$ yrs: both clumps are on 
roughly circular orbits at this time.

Evidently the clumps in both models are only marginally gravitationally bound and marginally tidally stable, as shown by the fact that they tend to disappear within an orbital period or less. Calculations with even higher spatial resolution have shown that the clumps become better-defined as a result (Boss 2005), suggesting that in the continuum limit, the clumps should survive to become protoplanets. An adaptive mesh refinement code will be needed to properly investigate the long-term survival of such clumps.

Figures 9 and 10 display the midplane temperature distributions for models FL1 and TE at the same times as the density distributions shown in Figures 7 and 8. The distributions are again highly similar, at least in the outer disk and in the clump-forming region. However, the region of the model FL1 disk inside about 6 AU does appear to be considerably more nonaxisymmetric than in the case of model TE, which is very nearly axisymmetric inside 6 AU. Evidently use of the flux-limiter can lead to significantly stronger nonaxisymmetric variations in the temperature field. However, these temperature changes have little effect on the clump-forming region of the disk, as the inner disk is the hottest region of the disk, with the midplane temperature rising to over $630 \mathrm{~K}$ at the inner boundary at $4 \mathrm{AU}$, sufficiently high to ensure gravitational stability $(Q>>1)$. Clumps do not form in the inner region in these models because of the high inner disk temperatures in the initial radial temperature profile.

In spite of the basic agreement after $\sim 8$ yrs of evolution, one must wonder what would happen on the much longer time scales that must be considered in deciding whether these clumps could survive to form gaseous protoplanets. In order to address this question, Figures 11 and 12 show the time evolutions of the volume-averaged midplane temperatures and total midplane thermal energies for both models. The intention is to discern if there are any trends evident over 8 yrs of evolution that could be used to decide the extent to which these two models might diverge if they could be evolved arbitrarily farther in time. Figures 11 and 12 reveal no such evidence for divergence: both of these quantities, when plotted for the entire midplane region (Figure 11), or only for the region from 6.5 to $13 \mathrm{AU}$ of most interest for disk instability (Figure 12), show that the two models evolve in very similar manners and give no hint that their evolutions might turn out to be significantly different if evolved even further in time.

The models with the flux-limiter run considerably slower than models without a fluxlimiter, as in order to maintain a stable solution of the energy equation with explicit time differences, a smaller time step (often $1 \%$ of the Courant time step) had to be employed. This fact is evident from Figures 11 and 12, which plot disk quantities every 10,000 time steps for models FL1 and TE: it is clear from the density of plot symbols that model FL1 
required many more time steps to evolve for the same period of time as model TE. The flux-limiter models each required roughly one year of machine time on a dedicated Alpha workstation to run for only up to 8 yrs of model evolution time, i.e., the models were being calculated only eight times faster than the disks were evolving in model time, a situation similar to current weather prediction models.

Rather than attempt to run these models significantly further in time, then, one can address the question of the extent to which the flux-limiter is having a long-term effect on the disk by examining more closely the evolution of the innermost disk, where the shorter orbital periods mean that the calculation has effectively been evolved for more dynamical times, i.e., for closer to a full orbital period. In order to be more quantitative than is possible by presenting only density and temperature contour plots, Figure 13 shows the amplitudes of the $m=1$ mode in the spherical harmonic representation of the midplane density distribution, as a function of radial distance, for models FL1 and TE. The time shown in Figure 13 was chosen in order to be as late as possible in the evolution of model FL1, yet as close as possible in time to model TE (data files are only stored every 10,000 times steps, so the times available for cross-comparison are quite limited as a result.) Figure 13 shows that the amount of nonaxisymmetry in the two models is nearly identical in the clumpforming region and beyond (outer $2 / 3$ in radius), but is still reasonably well-correlated even in the innermost disk. At some radii, model FL1 has a higher $m=1$ amplitude than model $\mathrm{TE}$, and the opposite is true at other inner disk radii. Figure 13 shows that the degree of nonaxisymmetry is well-correlated in both models, even in the innermost disk where orbital periods are the shortest, suggesting that the innermost disk shows little or no tendency for diverging in behavior, at least over these time scales, as a result of the flux-limiter.

Finally, Figures 14 and 15 show the effects of the flux-limiter on the convective energy fluxes in models FL1 and TE at $\sim 330$ yrs. The vertical convective energy flux is calculated as in Boss (2004) as the product of the local vertical velocity, cell area, specific internal energy, and cell density. Figures 14 and 15 plot this flux for a conical surface at a fixed angle of 0.3 degrees above the disk's midplane (i.e., the $J=2$ cells in the $\theta$ coordinate). The convective flux must vanish at the midplane as a consequence of the assumed equatorial symmetry of the models; if this constraint were to be lifted, more vigorous convective fluxes are to be expected (e.g., Ruden et al. 1988). These two figures show that application of the flux-limiter has no obvious systematic effects on the vertical convective fluxes near the midplane, where the need for convective cooling is most severe; the overall patterns of upwelling and downwelling regions are quite similar in both models. 


\section{Discussion}

These models have shown that the flux-limiter has relatively little effect on the evolution, at least during a phase when the disk has already begun forming strong spiral arms and clumps. The question arises as to what would happen if the flux-limiter was applied earlier in the evolution of the disk, prior to the formation of highly nonaxisymmetric structures. The model noted in passing by Boss (2001) began at an earlier time than in the present models, after 141 yrs of evolution instead of after $322 \mathrm{yrs}$, and so tested the effects of the flux-limiter at such an earlier phase. Unfortunately, the data files from the Boss (2001) fluxlimiter model no longer exist, as the models were run in 2000 and stored on a hard disk that has since failed. Hence it is not possible to present those results in the detail presented here, a fact that motivated calculation of the models in this paper. The flux-limiter model from Boss (2001) was compared to a non-flux-limiter model by visual inspection of density contour plots, as in Figures 1 and 2 and in Figures 7 and 8 in the present paper, with the conclusion being that there were no significant differences apparent in the degree of clumpiness in the two models. While purely a qualitative judgement, these results suggest that the role of the flux-limiter is similarly limited both early and late in the development of a phase of disk instability.

Boley et al. (2006, 2007b) have presented the results of a series of tests of their radiative hydrodynamics code on a "toy problem" (the plane-parallel grey atmosphere) with sufficient assumptions to permit an analytical solution for the temperature distribution. Their toy problem assumes an infinite slab, making the problem the same as a one-dimensional Cartesian atmosphere. This toy problem is well-suited to their cylindrical coordinate code, as their vertical $(z)$ cylindrical coordinate is effectively a one-dimensional Cartesian coordinate, and by freezing motion in the radial direction (Boley et al. 2006) and applying suitable boundary conditions at the disk edges the Boley et al. code can be used to simulate a plane-parallel atmosphere.

Boley et al. (2006) "... challenge all researchers who publish radiative hydrodynamics simulations to perform similar tests or to develop tests of their own and publish the results." While it would be ideal to be able to undertake the same tests as those examined by Boley et al. $(2006,2007 \mathrm{~b})$, the fact that their tests assume a plane-parallel atmosphere makes them unsuitable for a spherical coordinate code, which has no Cartesian coordinate. The closest analogue coordinate for the present code would be the $\theta$ coordinate, but trying to reproduce a plane-parallel atmosphere solution with spherical coordinates places the spherical coordinate code at a distinct disadvantage from the beginning, as any attempt to study a plane-parallel atmosphere with such a code will immediately introduce corrugations in all variables in each azimuthal $(r, \theta)$ plane. One could perhaps average over the entire disk to try to remove these 
corrugations, but the non-uniform $\theta$ grid spacing, designed to represent realistic protoplanetary disks, not plane-parallel slabs, would result in highly variable effective spacings in the vertical direction, which would further complicate the analysis. Studying the performance of the current radiative hydrodynamics code on the Boley et al. (2006, 2007b) tests in an unbiased manner requires writing a new one dimensional radiative hydrodynamics code based on the same numerical assumptions as the present three dimensional spherical coordinate code. Writing and testing such a code, even before trying the Boley et al. (2006, 2007b) tests, is a non-trivial task, as no such code exists. Writing such a code to perform the Boley et al. (2006, 2007b) tests would be a worthy goal for future work.

Alternatively, it is possible that a spherical coordinate version of the Boley et al. (2006, 2007b) tests could be posed and examined with the one dimensional spherical coordinate

version of the present code. This would also meet the request by Boley et al. (2006) "... to develop tests of their own and publish the results." This task remains for future investigation.

Finally, it should be noted that the motivation of this paper is the same as that expressed in the Boley et al. (2006) request "... to develop tests of their own and publish the results." Many other numerical tests of the present code have been presented as follows: spatial resolution (Boss 2000, 2005); gravitational potential solver (Boss 2000, 2001, 2005), artificial viscosity (Boss 2006a); and radiative transfer (Boss 2001, 2007). It would be valuable for other reseachers to consider their own tests of all of these key numerical aspects.

\section{Conclusions}

The results presented here confirm the statement made by Boss (2001) that the inclusion of a flux-limiter in these calculations does not lead to significantly different outcomes for the progress of a disk instability calculation. Even with the steeper vertical temperature gradient near the disk surface when a flux-limiter was employed (Figures 7 and 8), the corresponding midplane temperature increased by no more than $2 \%$. Similarly, Boss (2007) investigated the effects of several other changes in the treatment of radiative transfer in these models, finding that the numerical assumption that had the largest effect was the relaxation of the monotonically declining vertical temperature profile, which resulted in clumps that were no more than a factor of 2 times less dense than when monotonicity was enforced. For comparison, for models FL1 and TE in Figure 7 and 8, the maximum clump densities differed by only $25 \%$, implying even less of a difference between models FL1 and TE and the two models (H and TZ) from Boss (2007).

Evidently disk instability is tolerant of a range of treatments of the radiative transfer, at 
least up to a point. If there is a means for a clump to cool enough to contract, the clump will find this means to allow its survival. In this context it is of interest to note that analytical evaluations of disk instability (e.g., Rafikov 2007) have been restricted to considering plane parallel (one dimensional) disk models, where the entire disk midplane must be cooled, in order to cool the disk midplane anywhere at all. In a more realistic three dimensional disk model, of the sort depicted in the present numerical models, only the limited midplane region inside the dense clump needs to lose thermal energy, in any direction, in order for the clump to continue to contract and possibly survive to become a gas giant planet. This is a considerably relaxed criterion for cooling and ultimate clump survival compared to the cooling of an entire slab of midplane gas and dust. Similarly, a hot spot on the disk surface above a contracting clump will find it easier to radiate away its thermal energy than if the entire disk surface has the same vertical thermal profile as that under the hot spot.

Given the apparent observational need for disk instability to be able to form gas giant planets in some protostellar environments, if flux-limiters and other radiative transfer effects are not the main reason for the discrepant outcomes in models of disk instability, then there must be other reasons, or combinations of reasons, for these differences, as examined and discussed in some detail by Nelson (2006) and Boss (2007). Spurious heating of the inner disk associated with numerical oscillations is one possible source of these discrepancies that deserves further scrutiny (Boley et al. 2006, 2007b), as this leads to gravitational stability in the same region of the disk where clumps form in other disk instability models (Boss 2007).

Because of the unsatisfactory nature of the theoretical understanding of disk instabilities at present, it is important to continue to undertake code tests. The present models have shown that the use of a flux-limiter has relatively little effect on the evolution of an instability during the phase when the disk is already dynamically unstable. However, it is also important to investigate the role of a flux-limiter during earlier phases of evolution, before the disk becomes unstable, in order to learn if the flux-limiter can affect clump formation if applied from the very beginning of the evolution. A new model is underway that investigates this possibility, and the results will be presented in a future paper. Other code tests should also be sought, similar to the radiative transfer tests advanced by Boley et al. (2006, 2007b), except for spherical geometry instead of slab geometry, so that the present code can be tested in a similar manner.

I thank the referee for a number of good ideas for improving the manuscript, and Sandy Keiser for computer systems support. This research was supported in part by NASA Planetary Geology and Geophysics grants NNG05GH30G and NNX07AP46G, and is contributed in part to NASA Astrobiology Institute grant NCC2-1056. The calculations were performed on the Carnegie Alpha Cluster, the purchase of which was partially supported by NSF Major 
Research Instrumentation grant MRI-9976645.

\section{REFERENCES}

Alibert, Y., et al. 2005, A\&A, 434, 343

Andrews, S. M., \& Williams, J. P. 2007, ApJ, 659, 705

Bodenheimer, P., Yorke, H. W., Różyczka, M., \& Tohline, J. E. 1990, ApJ, 355, 651

Boley, A. C., et al. 2006, ApJ, 651, 517

Boley, A. C., Hartquist, T. W., Durisen, R. H., \& Michael, S. 2007a, ApJ, 656, L89 (erratum 660, L175)

Boley, A. C., Durisen, R. H., Nordlund, A., \& Lord, J. 2007b, ApJ, 665, 1254

Boss, A. P. 1984, ApJ, 277, 768

—. 1997, Science, 276, 1836

- 2000, ApJ, 536, L101

- 2001, ApJ, 563, 367

—. 2002, ApJ, 567, L149

- 2004, ApJ, 610, 456

- 2005, ApJ, 629, 535

—. 2006a, ApJ, 641, 1148

—. 2006b, ApJ, 643, 501

—. 2007, ApJL, 661, L73

Boss, A. P., \& Myhill, E. A. 1992, ApJS, 83, 311

Cai, K., et al. 2006, ApJL, 636, L149 (erratum 642, L173)

Chambers, J. E. 2006, ApJ, 652, L133

Chick, K. M., \& Cassen, P. 1997, ApJ, 477, 398

Cieza, L., et al. 2007, ApJ, 667, 308

Cochran, W. D., Endl, M., Wittenmyer, R. A., \& Bean, J. L. 2007, ApJ, 665, 1407

Ikoma, M., et al. 2006, ApJ, 650, 1150

Inaba, S., Wetherill, G. W. \& Ikoma, M. 2003, Icarus, 166, 46

Kitamura, Y., et al. 2002, ApJ, 581, 357 
Kornet, K., Wolf, S., \& Różyczka, M. 2006, A\&A, 458, 661

Laughlin, G., Bodenheimer, P., \& Adams, F. C. 2004, ApJ, 612, L73

Levermore, C. D., \& Pomraning, G. C. 1981, ApJ, 248, 321

Mayer, L, Quinn, T., Wadsley, J., \& Stadel, J. 2002, Science, 298, 1756

—. 2004, ApJ, 609, 1045

Mayer, L, Lufkin, G., Quinn, T., \& Wadsley, J. 2007, ApJL, 661, L77

Mizuno, H. 1980, Prog. Theor. Phys., 64, 544

Myhill, E. A., \& Boss, A. P. 1993, ApJS, 89, 345

Nelson, A. F. 2006, MNRAS, 373, 1039

Pickett, B. K., Cassen, P., Durisen, R. H., \& Link, R. 2000, ApJ, 529, 1034

Rafikov, R. R. 2007, ApJ, 662, 642

Ruden, S. P., Papaloizou, J. C. B., \& Lin, D. N. C. 1988, ApJ, 329, 739

Sato, B., et al. 2005, ApJ, 633, 465

Sigurdsson, S., et al. 2003, Science, 301, 193

Whitehouse, S. C., \& Bate, M. R. 2006, MNRAS, 367, 32 


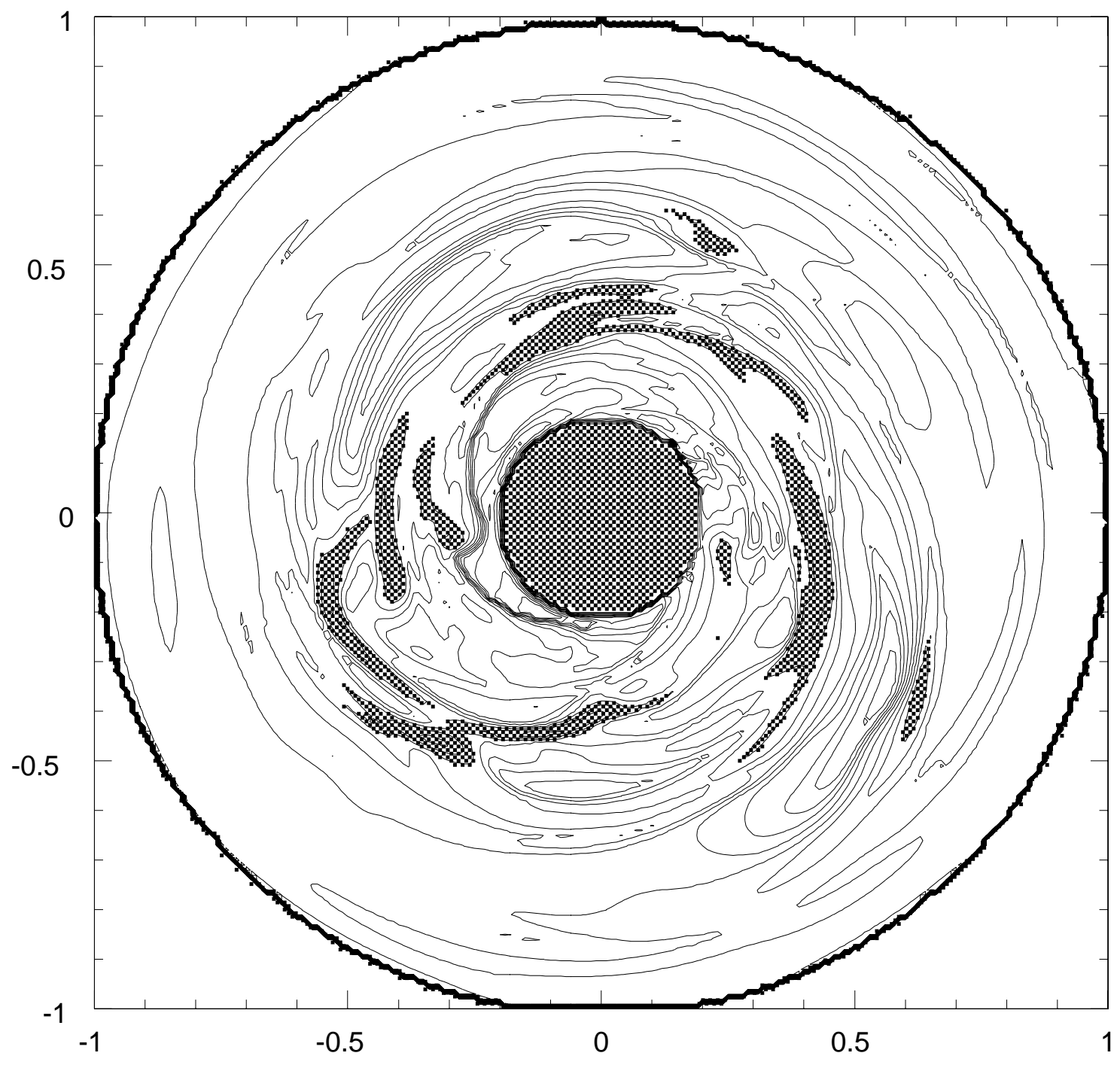

Fig. 1.- Equatorial density contours for model F1 after 325.8 yrs of evolution. The disk has an outer radius of $20 \mathrm{AU}$ and an inner radius of $4 \mathrm{AU}$. Hashed regions denote clumps and spiral arms with densities higher than $10^{-10} \mathrm{~g} \mathrm{~cm}^{-3}$. Density contours represent factors of two change in density. 


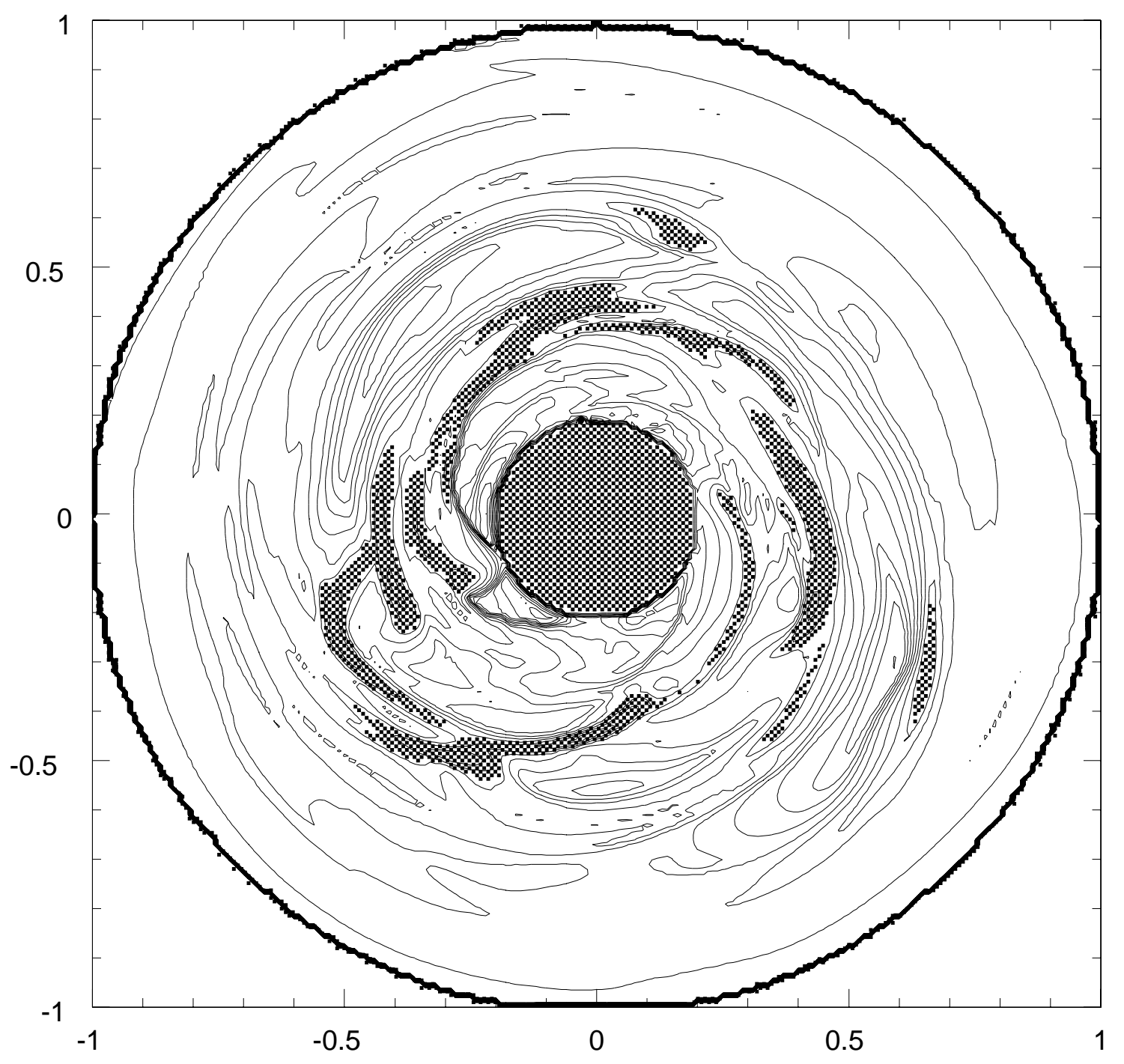

Fig. 2.- Same as Figure 1, but for model TE after 326.5 yrs. 


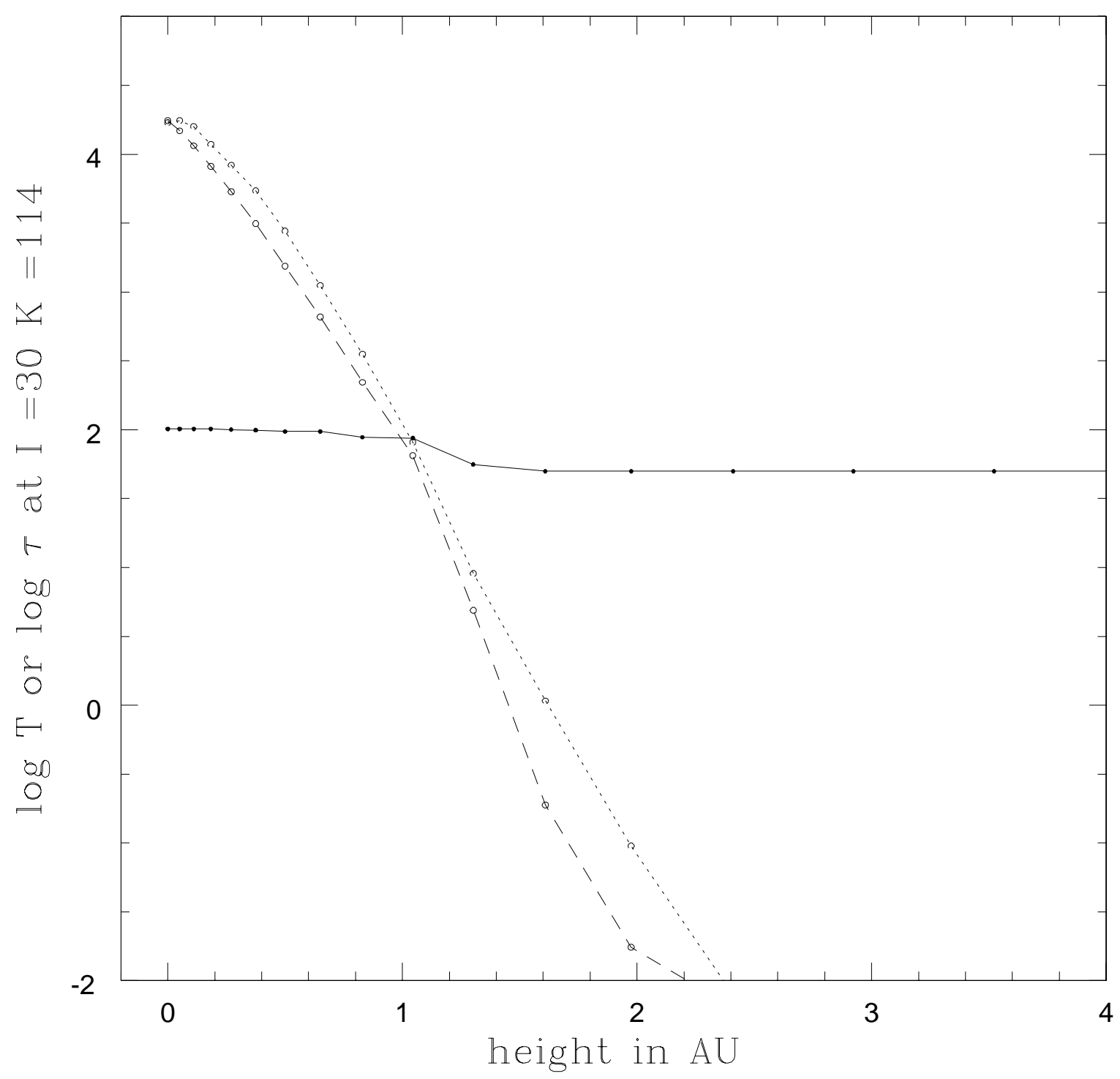

Fig. 3.- Log of the optical depth (dashed lines) and temperature (solid line) as a function of distance above the midplane for model FL1 after $325.8 \mathrm{yrs}$ of evolution. The profiles are along the $\theta$ coordinate, starting at the location of the maximum density in the midplane. The long-dashed line gives the optical depth in the $\theta$ direction, starting with zero at the rotational (symmetry) axis, while the short-dashed line gives the optical depth in the radial direction, starting with zero at the outer edge of the spherical computational volume. 


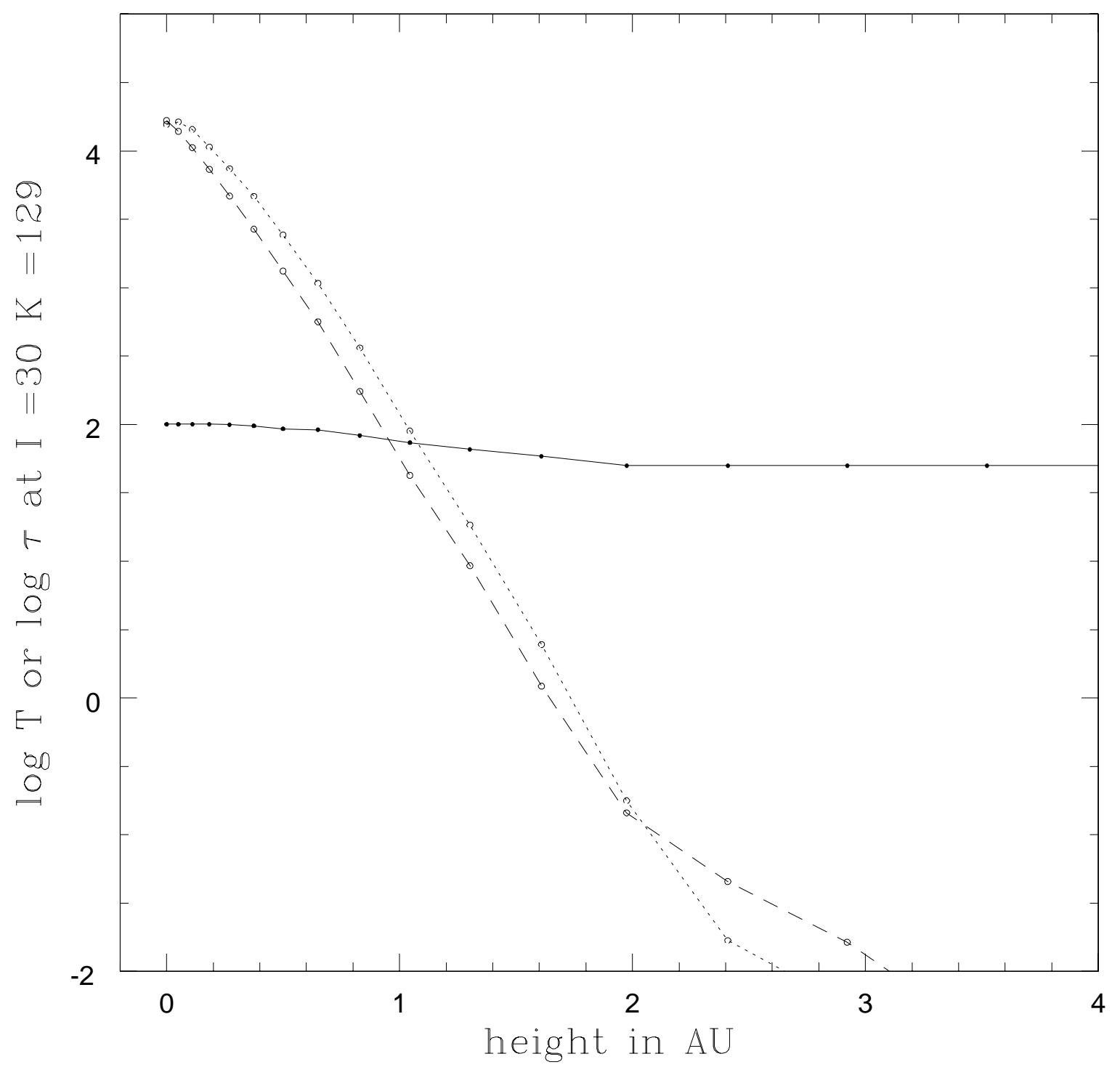

Fig. 4. - Same as Figure 3, but for model TE after 326.5 yrs. 


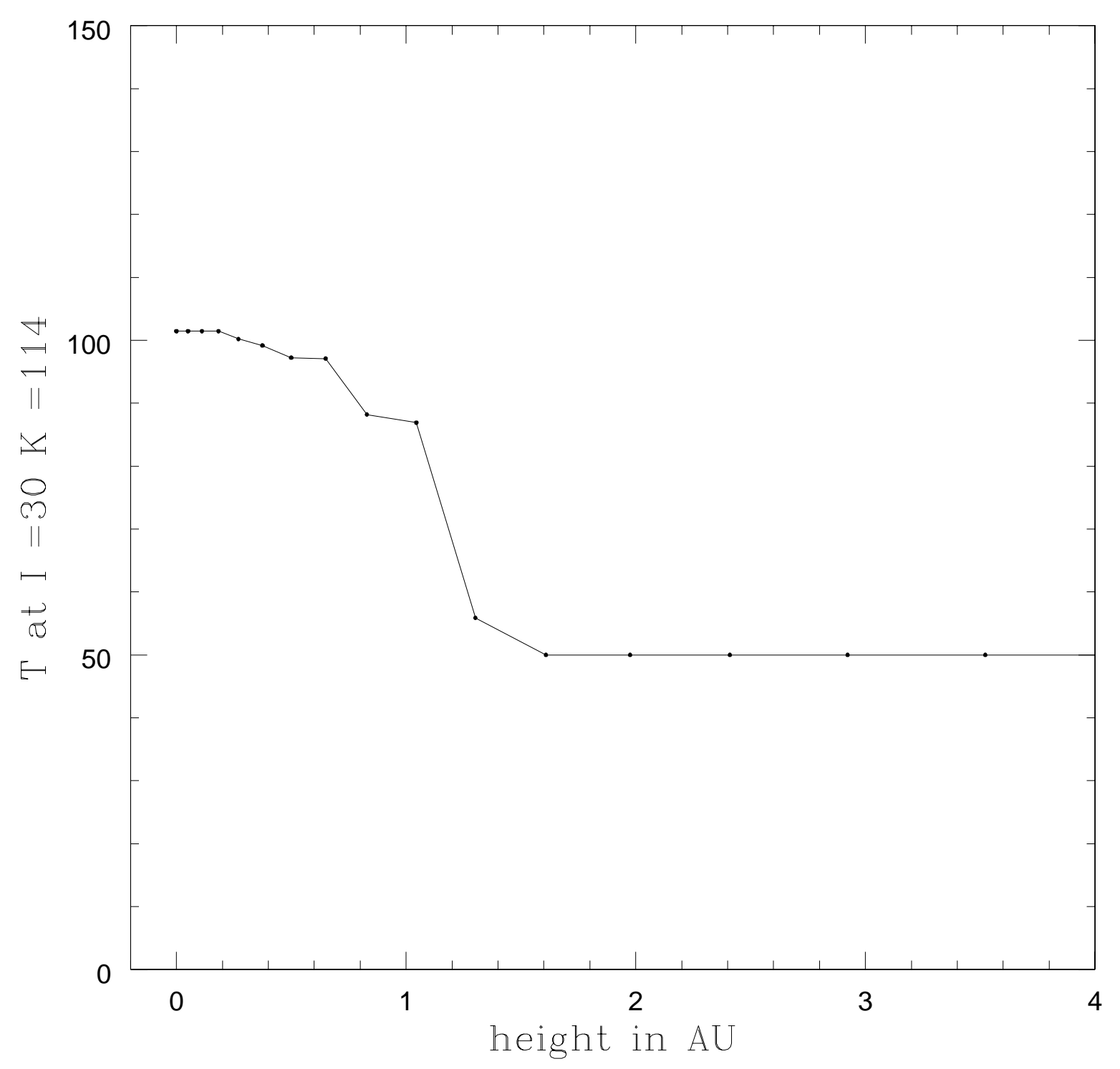

Fig. 5.- Temperature (linear scale) as a function of distance above the midplane for model FL1 after 325.8 yrs of evolution, plotted as in Figure 3. 


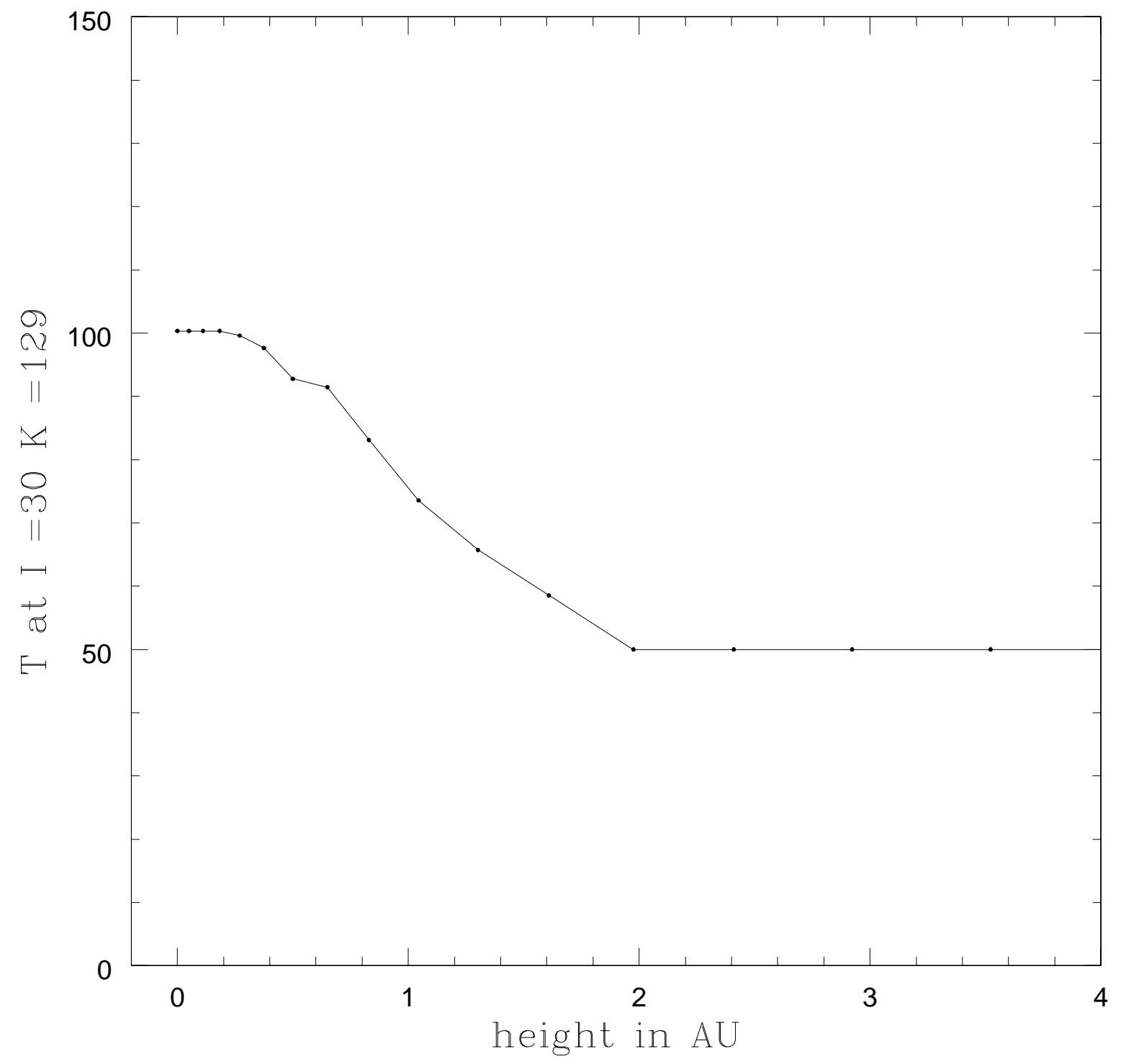

Fig. 6. - Same as Figure 5, but for model TE after 326.5 yrs. 


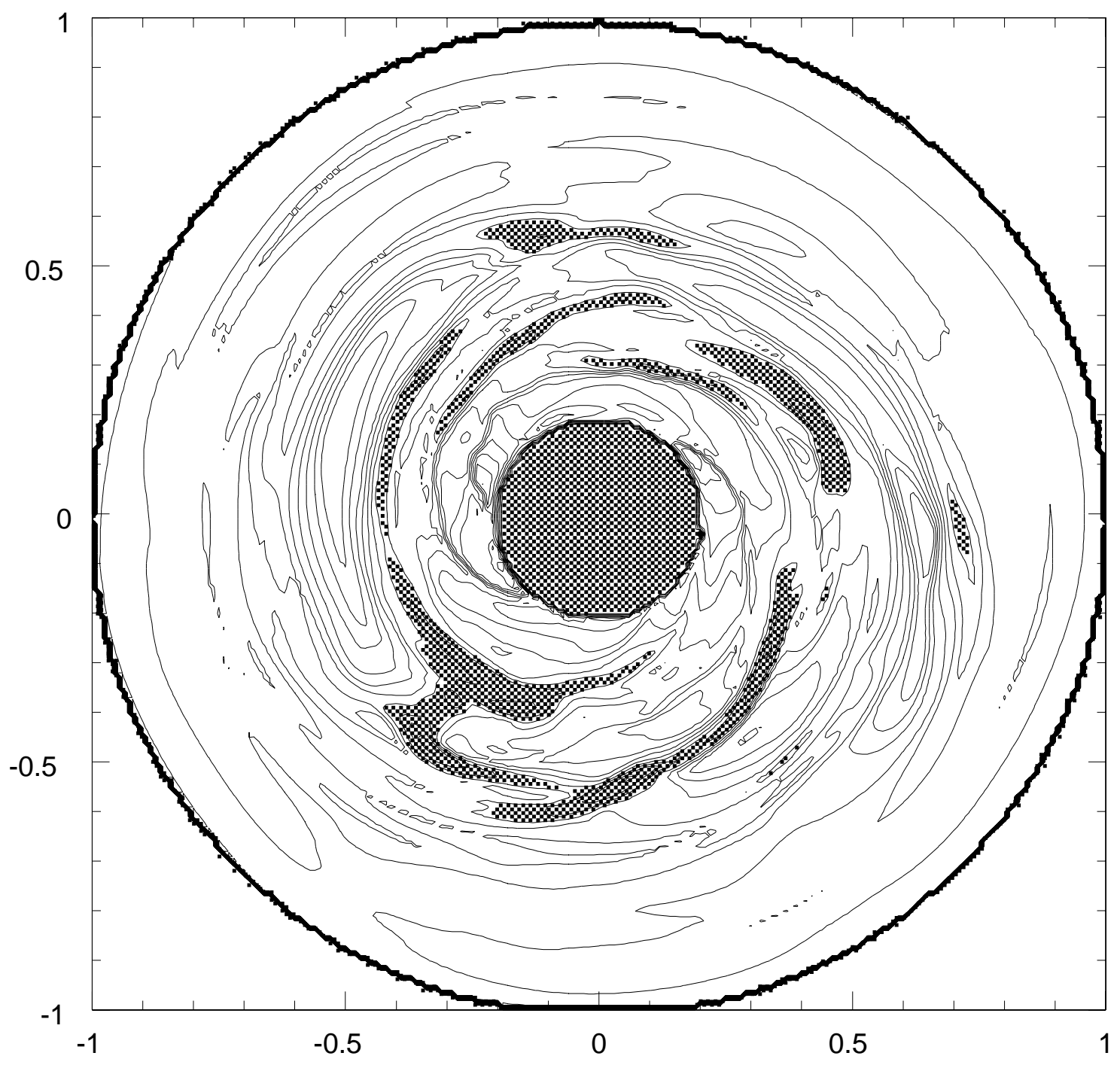

Fig. 7.- Equatorial density contours for model FL1 after 329.6 yrs of evolution, plotted as in Figure 1. 


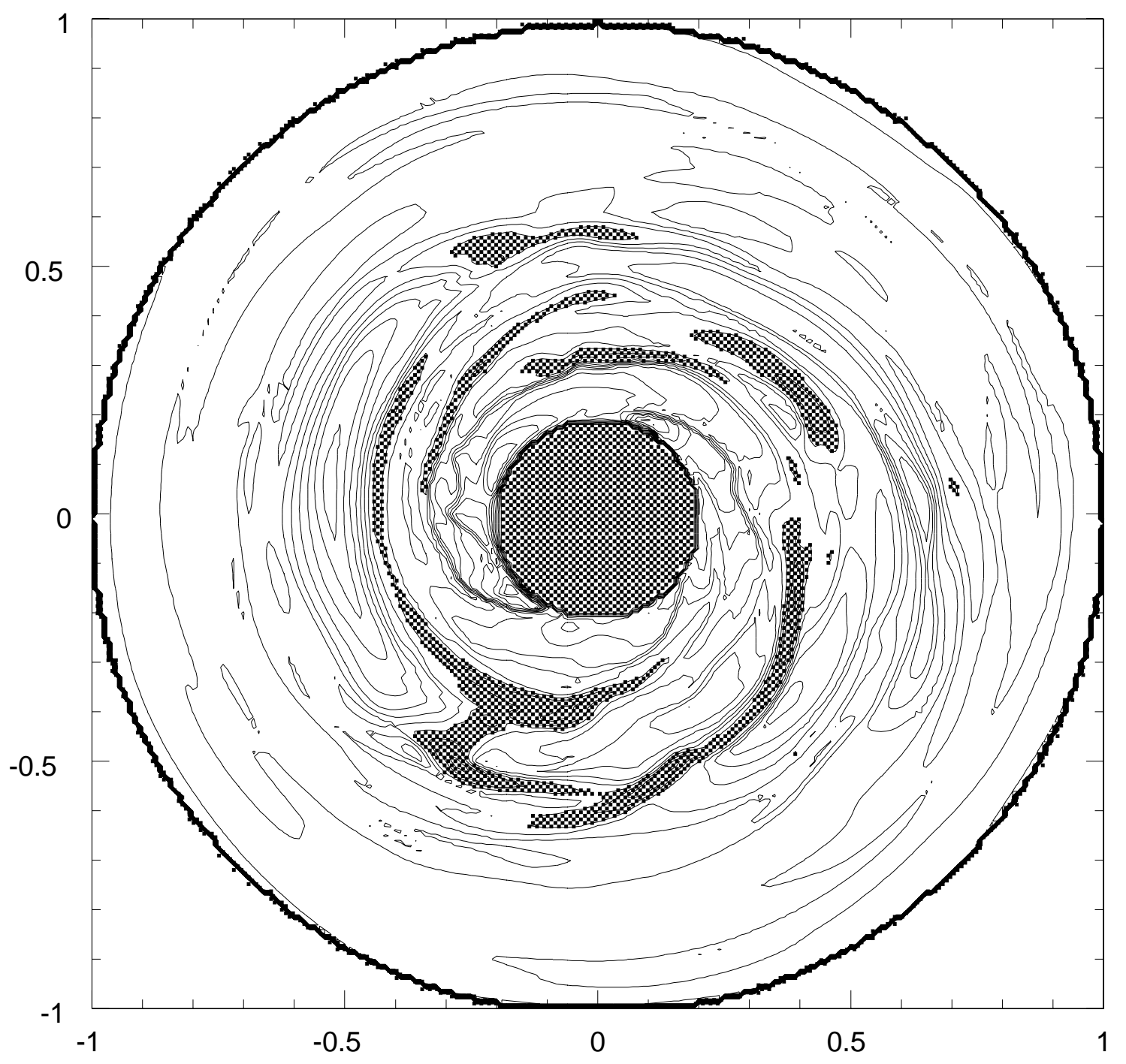

Fig. 8.- Same as Figure 7, but for model TE after 330.3 yrs. 


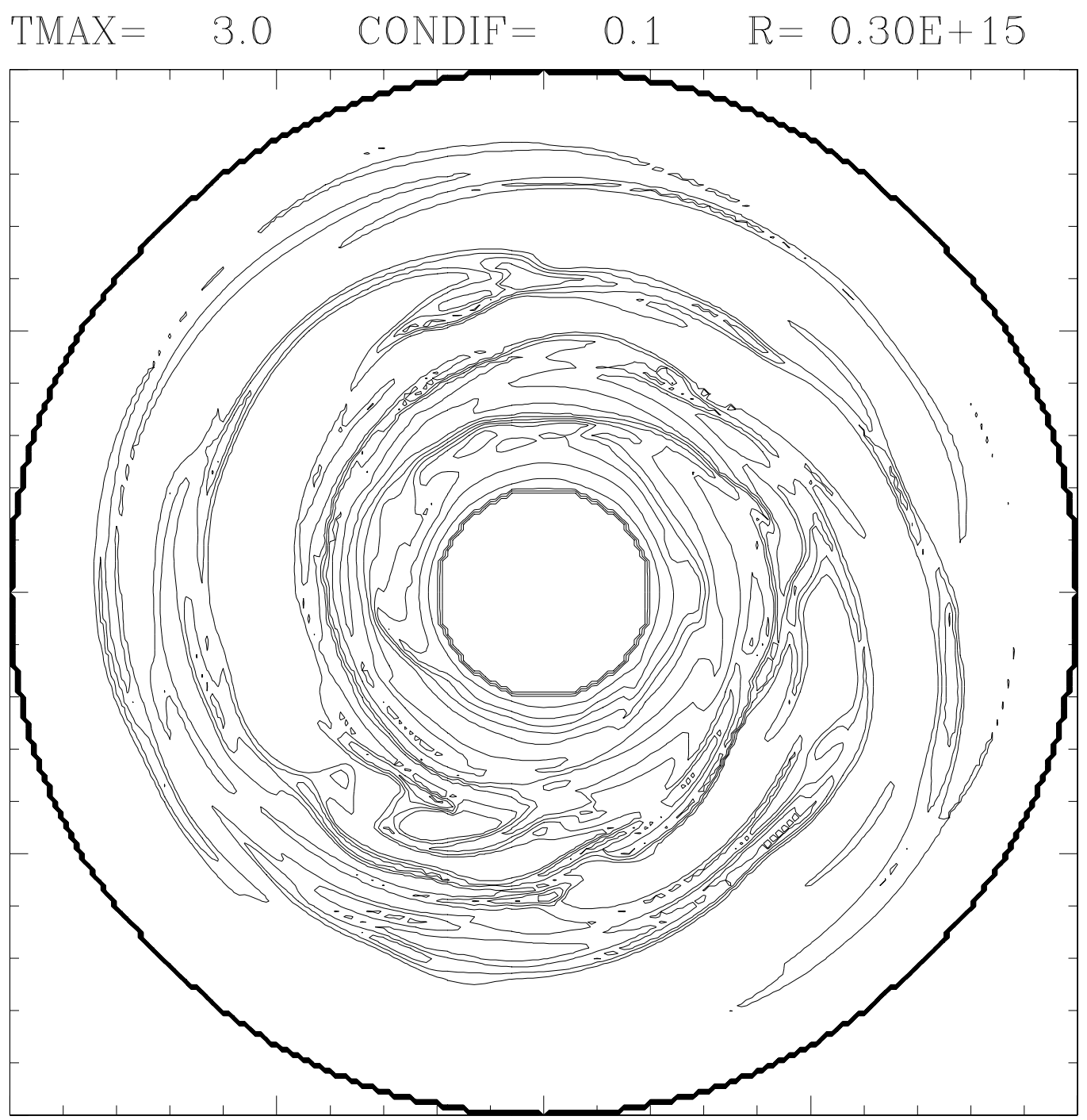

Fig. 9.- Equatorial temperature contours for model FL1 after 329.6 yrs of evolution, plotted as in Figure 1, with temperature contours representing factors of 1.26 changes in temperature. 


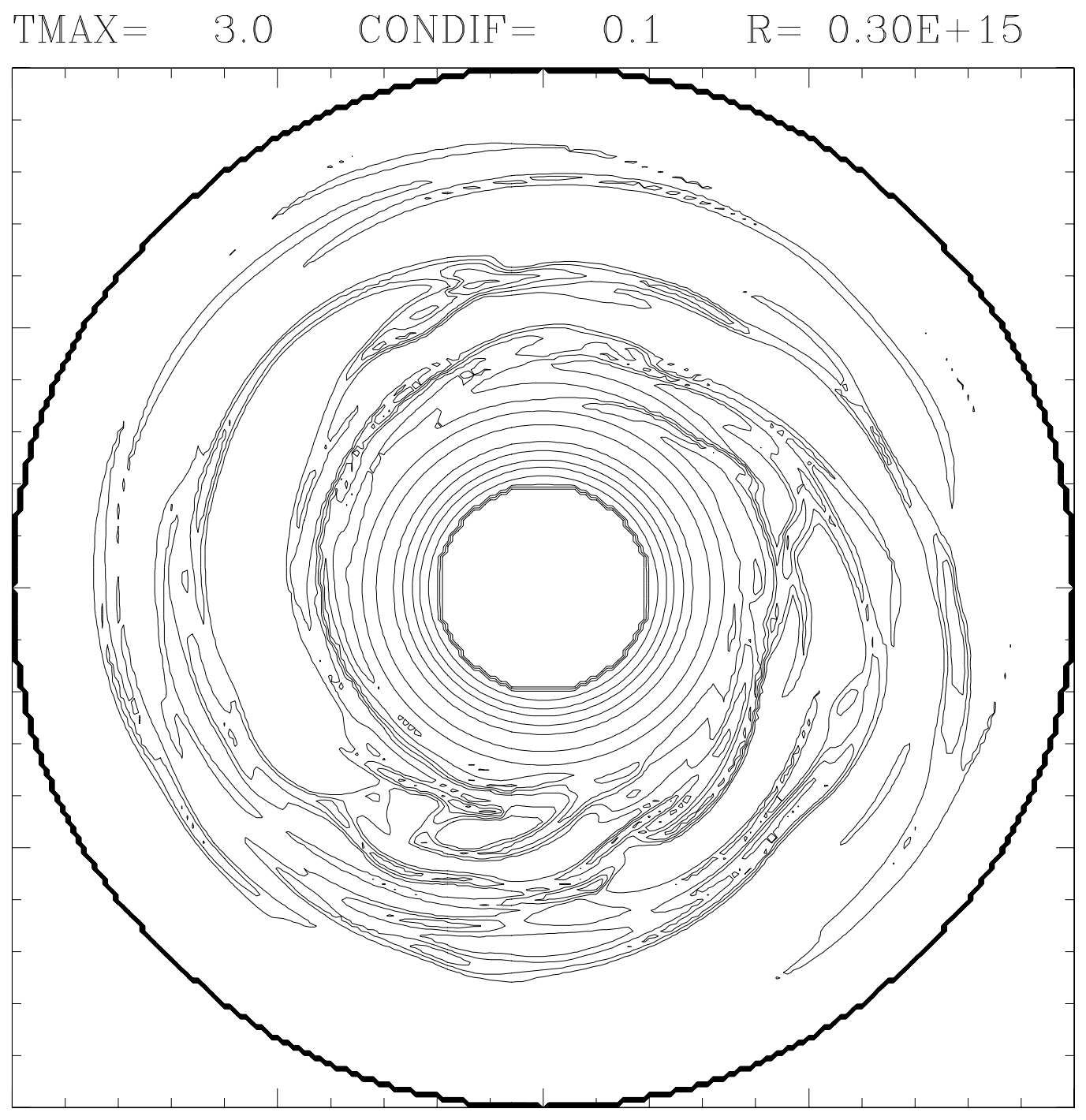

Fig. 10.- Same as Figure 9, but for model TE after 330.3 yrs. 


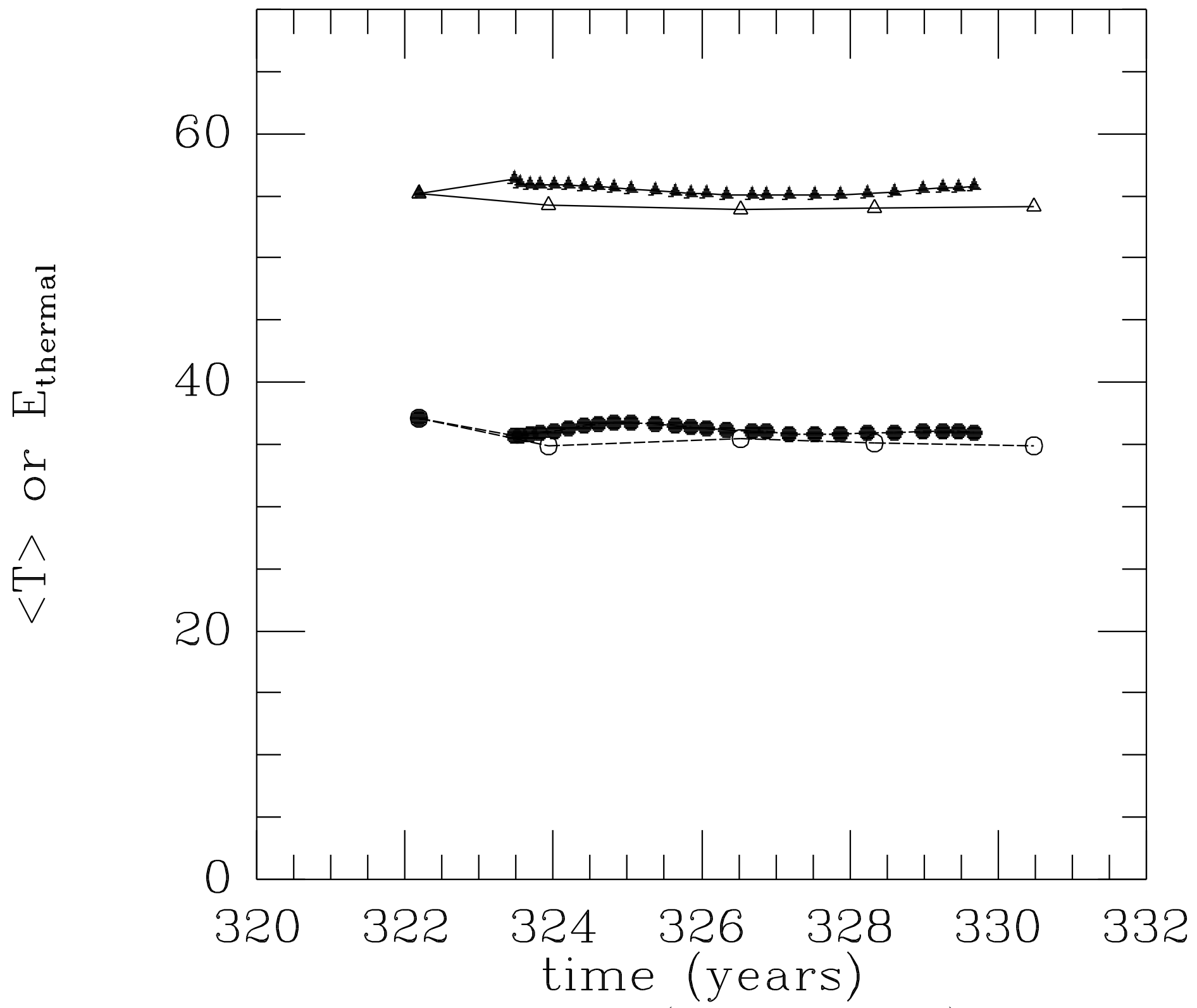

Fig. 11. - Volume-averaged midplane temperatures (triangles and solid lines) and total midplane $E_{\text {thermal }}$ (circles and dashed lines) for model FL1 (filled symbols) and model TE (open symbols) as a function of time in years. Temperatures are given in $\mathrm{K}$ and the total thermal energy in units of $10^{39}$ ergs. 


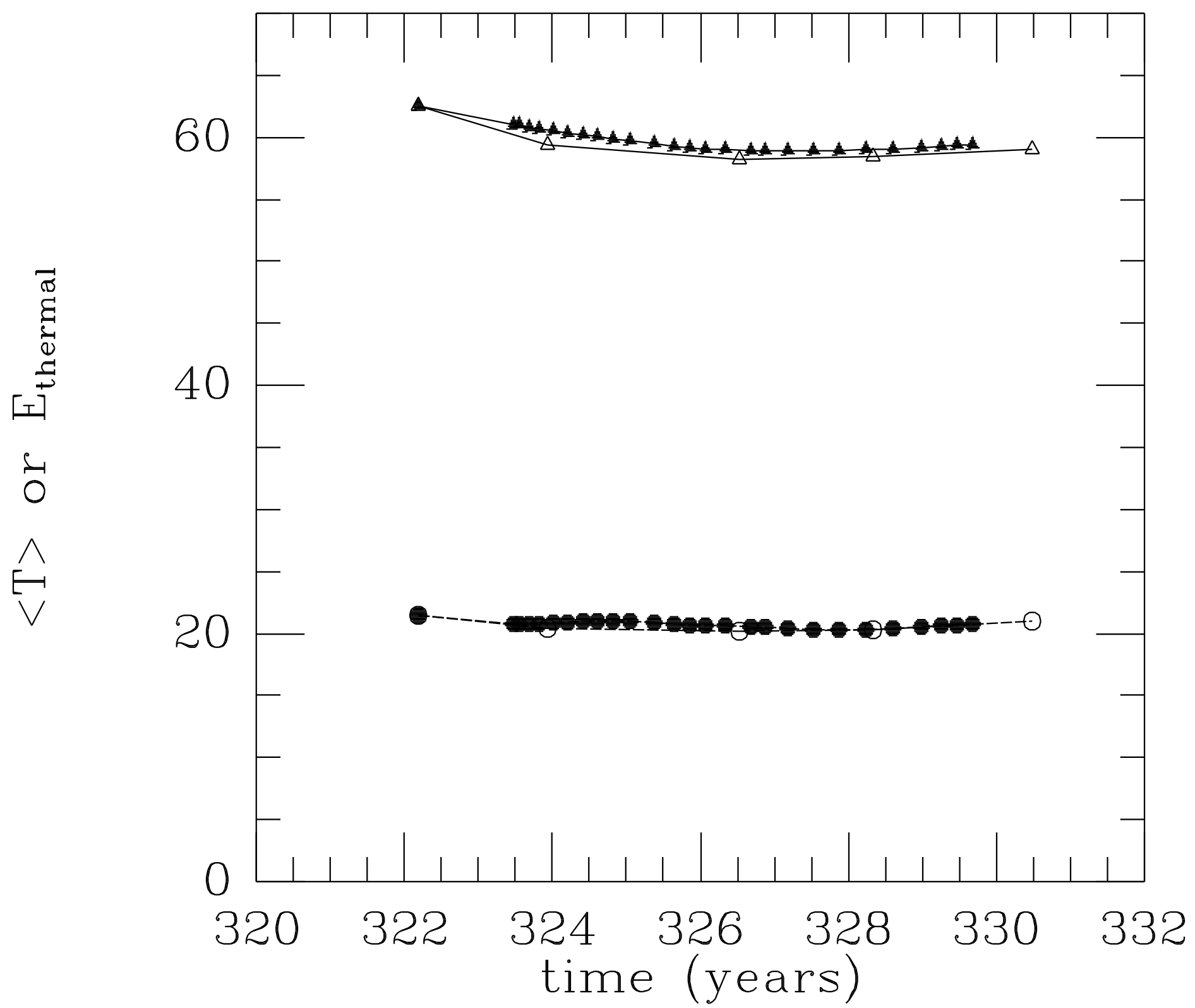

Fig. 12.- Same as Figure 11, but only for radial distances of 6.5 to 13 AU in the midplane. 


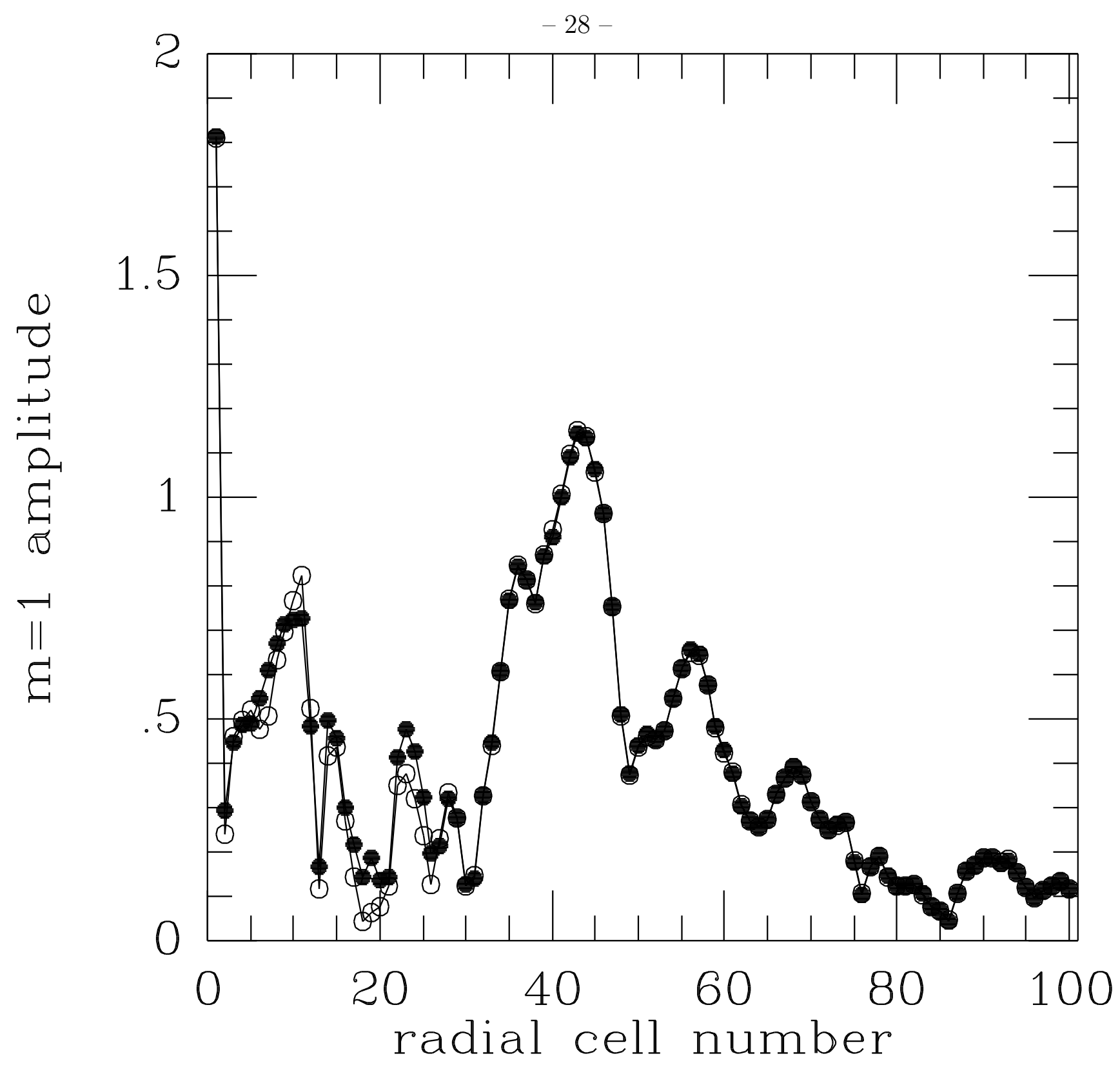

Fig. 13.- Amplitudes of the $m=1$ mode in a spherical harmonic expansion of the midplane density distribution as a function of radial distance in the disk, with radial cell number 1 located at $4 \mathrm{AU}$ and cell number 100 located at $20 \mathrm{AU}$. The amplitudes for model FL1 (filled symbols) and for model TE (open symbols) are shown at 328.2 and 328.3 yrs, respectively. 


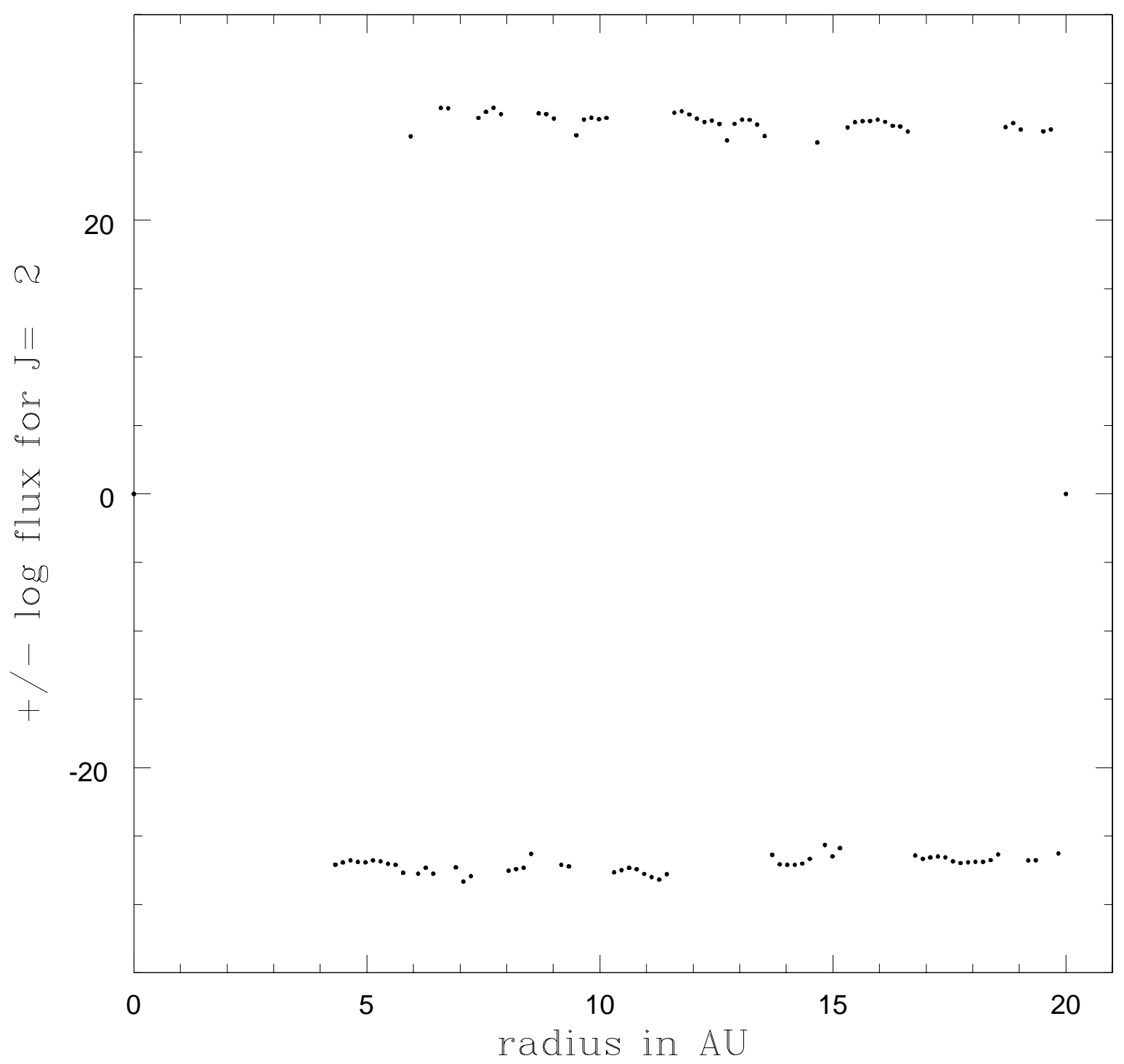

Fig. 14. - Logarithm of the vertical convective flux (cgs units) as a function of radial distance for model FL1 at 329.6 yrs. Values are plotted for a conical surface 0.3 degrees above the midplane, where the fluxes must vanish. Positive fluxes refer to upward transport, while negative fluxes correspond to downward transport. 


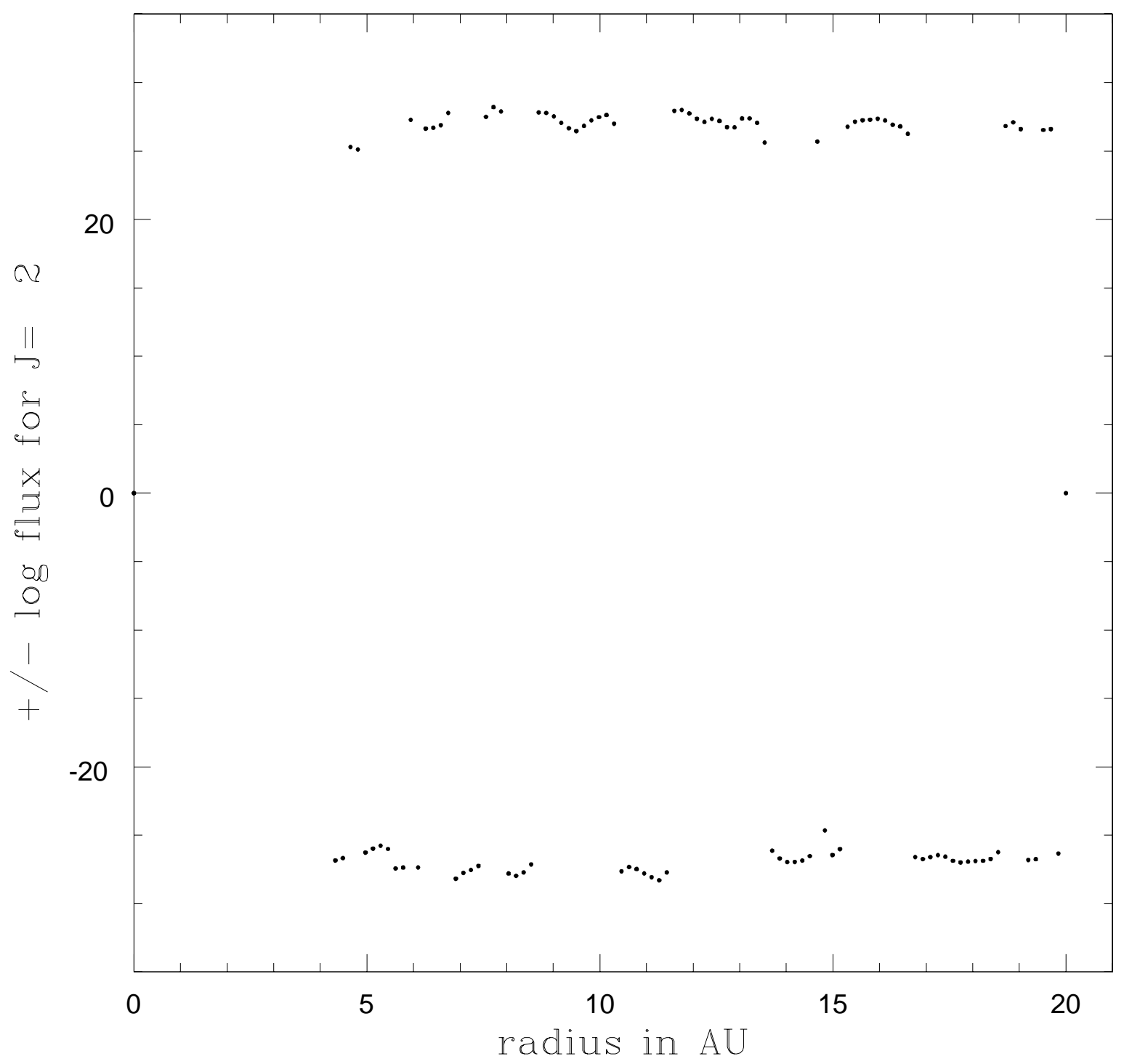

Fig. 15.- Same as Figure 14, but for model TE at 330.3 yrs. 